\title{
An attempt to identify the extended synchrotron structure associated with the micro-quasar GRS $1915+105$
}

\author{
M. Ostrowski ${ }^{1}$ and E. Fürst ${ }^{2}$ \\ 1 Obserwatorium Astronomiczne, Uniwersytet Jagielloński, ul.Orla 171, 30-244 Kraków, Poland \\ 2 Max-Planck-Institut für Radioastronomie, Auf dem Hügel 69, 53-201 Bonn 1, Germany \\ Received 18 October 2000 / Accepted 6 November 2000

\begin{abstract}
The energy ejected from the galaxy micro-quasar GRS 1915+105 in the form of jets is expected to lead to formation of an extended double lobe/hot-spot structure with an energy content comparable to an average supernova remnant. We used the Effelsberg $100 \mathrm{~m}$ telescope at $10.45 \mathrm{GHz}$ in attempt to identify such structures. For this distant galactic plane source, no definite identification was possible, due to high confusion by numerous background sources; however, a few suspect structures were identified.
\end{abstract}

Key words. ISM: jets and outflows - shock waves - acceleration of particles - radio continum: ISM

\section{Introduction}

Among seven recently known galactic sources of relativistic jets (cf. Mirabel \& Rodríguez 1999), the object GRS 1915+105, a first discovered galactic micro-quasar, is in some respects the most interesting. From the X-ray binary harbouring a black hole candidate, jets are occasionally ejected with a velocity $V_{\mathrm{j}} \approx 0.92 c$, as measured with the VLA (Mirabel \& Rodríguez 1994), or even reaching $\approx 0.98 c$, as derived from MERLIN observations at smaller spatial scales (Fender et al. 1999). In several years of observations a recurrent activity of the source was monitored (cf. Fender \& Pooley 1998; Rodríguez \& Mirabel 1999; Yadav et al. 1999).

With the estimated mass of ejected material of $210^{25} \mathrm{~g}$, Mirabel \& Rodríguez (1994) derived a large value for the kinetic energy in the ejected components' bulk motion, $E_{\mathrm{k}}=310^{46} \mathrm{erg}$. They noted that the power of the process ejecting blobs of radio emitting plasma is $\sim 400$ times greater than the steady $\mathrm{X}$-ray flux radiated at the same time. A detailed discussion of the energy output in the form of jets from this source (cf. Levinson \& Blandford 1996; Ghisellini 1999; Gliozzi et al. 1999; Rodríguez \& Mirabel 1999) shows that varying assumptions about ejected particles and the jet Poynting flux can substantially scale down this energy estimate for a single ejected blob, but the average kinetic power injected into the medium in the form of jets can be larger than the maximum steady photon luminosity of $\sim 310^{38} \mathrm{erg} / \mathrm{s}$. Additionally, Fender \& Pooley (2000)

Send offprint requests to: M. Ostrowski,

e-mail: mio@oa.uj.edu.pl suggest that GRS $1915+105$ can inject more energy and matter into the outflow during periods of repeated small events than it does during the large ejections. Thus, the source activity, expected to last at least several $10^{5}$ years, will pump into the surrounding medium energy equal to at least $\sim 10^{51} \mathrm{ergs}$, an amount comparable to the energy injected by an average supernova event. It is expected that processes dissipating this energy - shock waves and the generation of turbulent plasma motions - generate also populations of energetic electrons, which in turn may be observed through their synchrotron radiation. As a final result, formation of a large scale radio source with a classical double structure is expected, presumably similar to extragalactic FRII sources. As estimated by Levinson \& Blandford (1996) the source can reach a spatial extension of up to a few hundred parsecs. For the estimated GRS $1915+105$ distance near $12 \mathrm{kpc}$ and the angle of jet ejections with respect to the line of sight close to $70^{\circ}$, the angular extension of the respective extended source may reach $\sim 1^{\circ}$ on the sky.

A few objects among strong binary X-ray sources were discovered as radio stars, with small scale radio jets (cf. Hjellming 1988; Mirabel \& Rodríguez 1999). Among them, only two exhibit extended diffuse structures. The relativistic jets source SS433, situated two degrees below the Galactic plane, is influencing (powering) a $1^{\circ} \times 2^{\circ}$ synchrotron nebula, which is assumed to be a supernova remnant. The second, Circinus X-1, is at the Galactic plane and is surrounded by the 20 arc min synchrotron source, elongated along the small-scale jet direction. The source Scorpius X-1 is accompanied by two radio hot-spots at distances $\approx 1^{\prime}$. However, all these sources are much (a few 
orders of magnitude) weaker in X-rays and in their jet kinetic power than GRS 1915+105. Looking for analogies for GRS 1915+105, one could consider Cygnus X-3, a possible black hole candidate. In this source, relativistic ejections were observed at mili-arc-second scales, but there is no definite identification of a large scale structure associated with this object.

Rodríguez \& Mirabel (1998, 三 RM98) performed a search of extended counterparts of GRS 1915+105 with the use of VLA at $20 \mathrm{~cm}$ (configuration D) to look for possible hot spots at jets' terminal shocks. They discovered bright spots coincident with infra-red sources IRAS 19132+1035 and IRAS 19124+1106. Detailed observations of these sources at a few VLA frequencies $(20 \mathrm{~cm}$, $6 \mathrm{~cm}$ and $2 \mathrm{~cm}$ ) revealed a non-thermal feature extended along the jet position angle. Both sources were also observed with the use of H92 $\alpha$ recombination line. The results are fully compatible with HII regions in distances 6 and $7 \mathrm{kpc}$, and the mentioned non-thermal feature could be an unconfirmed indication of association with GRS $1915+105$. Also, a continuation of this work (Chaty et al. 2000) does not lead to a clear conclusion in this matter.

Independent from RM98, we performed a search of the above mentioned extended structure using the Effelsberg $100 \mathrm{~m}$ telescope at $10.45 \mathrm{GHz}$. As this instrument is more able to recognize diffuse synchrotron features, one can consider our observations as complimentary to the ones of RM98. Also, we considered a substantially larger region of $2^{\circ} \times 2^{\circ}$ near GRS $1915+105$. The performed observations did not provide any direct proof for the association of the observed synchrotron features with jets. With respect to RM98, two new more distant hot spots, coincident with the jet direction, are noted.

\section{Observations}

To plan new observations we analysed a $2^{\circ} \times 2^{\circ}$ region centred approximately at the position of GRS $1915+105$ $\left(\alpha(1950)=19^{\mathrm{h}} 12^{\mathrm{m}} 49.966^{\mathrm{s}}, \delta(1950)=10^{\circ} 51^{\prime} 26.73^{\prime \prime}\right)$, based on existing data. Figure 1 shows the superposition of the radio emission at an $11 \mathrm{~cm}$ wavelength, taken from the Effelsberg Galactic plane survey (Reich et al. 1984) on the IRAS $60 \mu \mathrm{m}$ emission (Beichmann et al. 1985). Two complexes of HII regions dominate by their strong emission $\left(\alpha(1950)=19^{\mathrm{h}} 11^{\mathrm{m}} 47^{\mathrm{s}}, \delta(1950)=11^{\circ} 07^{\prime} 03^{\prime \prime}, \alpha(1950)=\right.$ $19^{\mathrm{h}} 11^{\mathrm{m}} 06^{\mathrm{s}}, \delta(1950)=10^{\circ} 48^{\prime} 25^{\prime \prime}$, see Wink et al. 1982). The shell-type emission feature near $\alpha(1950)=19^{\mathrm{h}} 14^{\mathrm{m}}$, $\delta(1950)=11^{\circ} 4^{\prime}$ was identified as a supernova remnant by Fürst et al. (1987). Within a cone of jet position angles measured by various authors (cf. Fender et al. 1999; Rodríguez \& Mirabel 1999; Sams et al. 1996) the one $\left(\mathrm{PA}=155^{\circ}\right)$, accurately determined by Mirabel \& Rodríguez (1994), is indicated in the figure.

Along this line, three patchy emission features are visible, which have not yet been identified. They are denoted as $\mathrm{A}, \mathrm{B}$, and $\mathrm{C}$. We have conducted new observations of these three regions at $10.45 \mathrm{GHz}$ with the Effelsberg 100 -m-radiotelescope to identify the nature of the radio

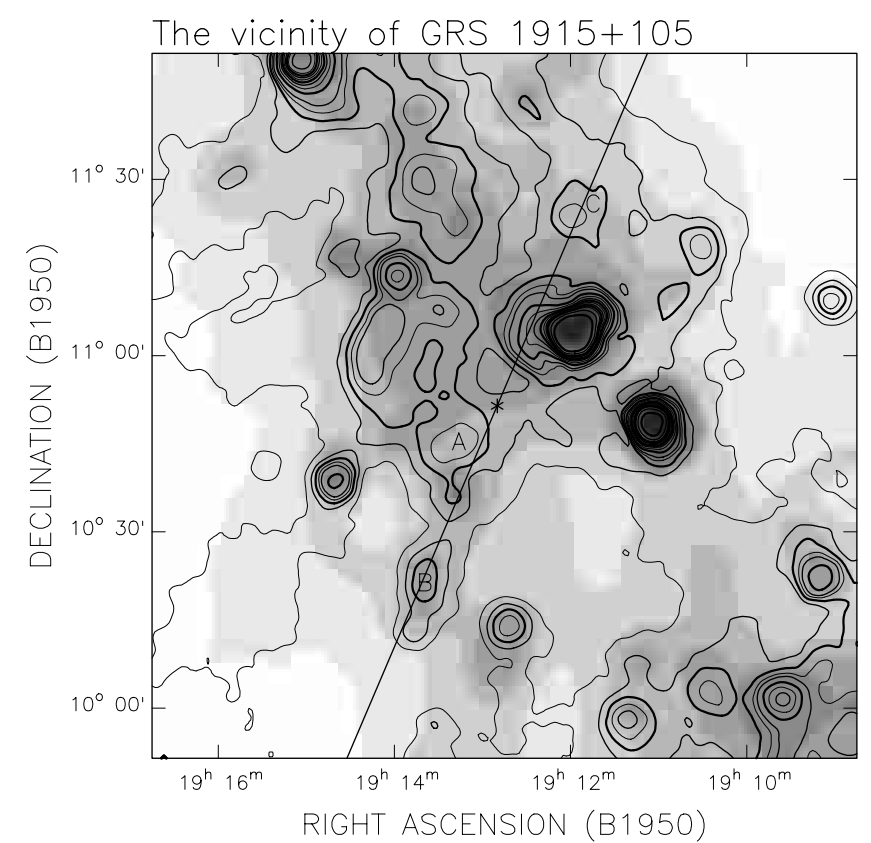

Fig. 1. Two square degree vicinity of GRS 1915+105. An 11-cm Bonn map (contours) is superposed on the IRAS infrared map at $60 \mu \mathrm{m}$ (shaded intensity scale). The position of GRS $1915+105$ is indicated with an asterisk, and the Mirabel \& Rodríguez (1994) jets' position with a solid line. Three regions observed by us are denoted with the symbols $\mathrm{A}, \mathrm{B}$, and C, respectively

Table 1. Parameters of the $10.45 \mathrm{GHz}$ observations

\begin{tabular}{ll}
\hline Date of observation & $25 \mathrm{January} 1998$ \\
Frequency & $10.45 \mathrm{GHz}$ \\
Bandwidth & $300 \mathrm{MHz}$ \\
Calibrator & $3 \mathrm{C} 286$ \\
Calibrator flux density & $4.5 \mathrm{Jy}$ \\
Calibrator polarization & $11.7 \%$ at $31^{\circ}$ \\
$\mathrm{HPBW}$ & $69^{\prime \prime}$ \\
$T_{\mathrm{B}} / S[\mathrm{~K} / \mathrm{Jy}]$ & 2.43 \\
Sensitivity I & $5.1 \mathrm{mKT}_{\mathrm{B}}$ \\
Sensitivity PI & $2.4 \mathrm{mKT}_{\mathrm{B}}$ \\
\hline
\end{tabular}

emission. We made use of the high-sensitivity four-feed receiver system to map the objects in total power and linear polarization. The observations were made by moving the telescope in azimuthal direction along the four feeds. The system uses the "software beam switching" technique (Morsi \& Reich 1988). The restoration procedure was introduced by Emerson et al. (1979). A detailed description of the system is given by Schmidt et al. (1993). Some parameters relevant for the current observation are summarized in Table 1.

The results for total power of the three regions are combined on one map and displayed in Fig. 2. In polarization, no signal beyond 3 rms was detected. 


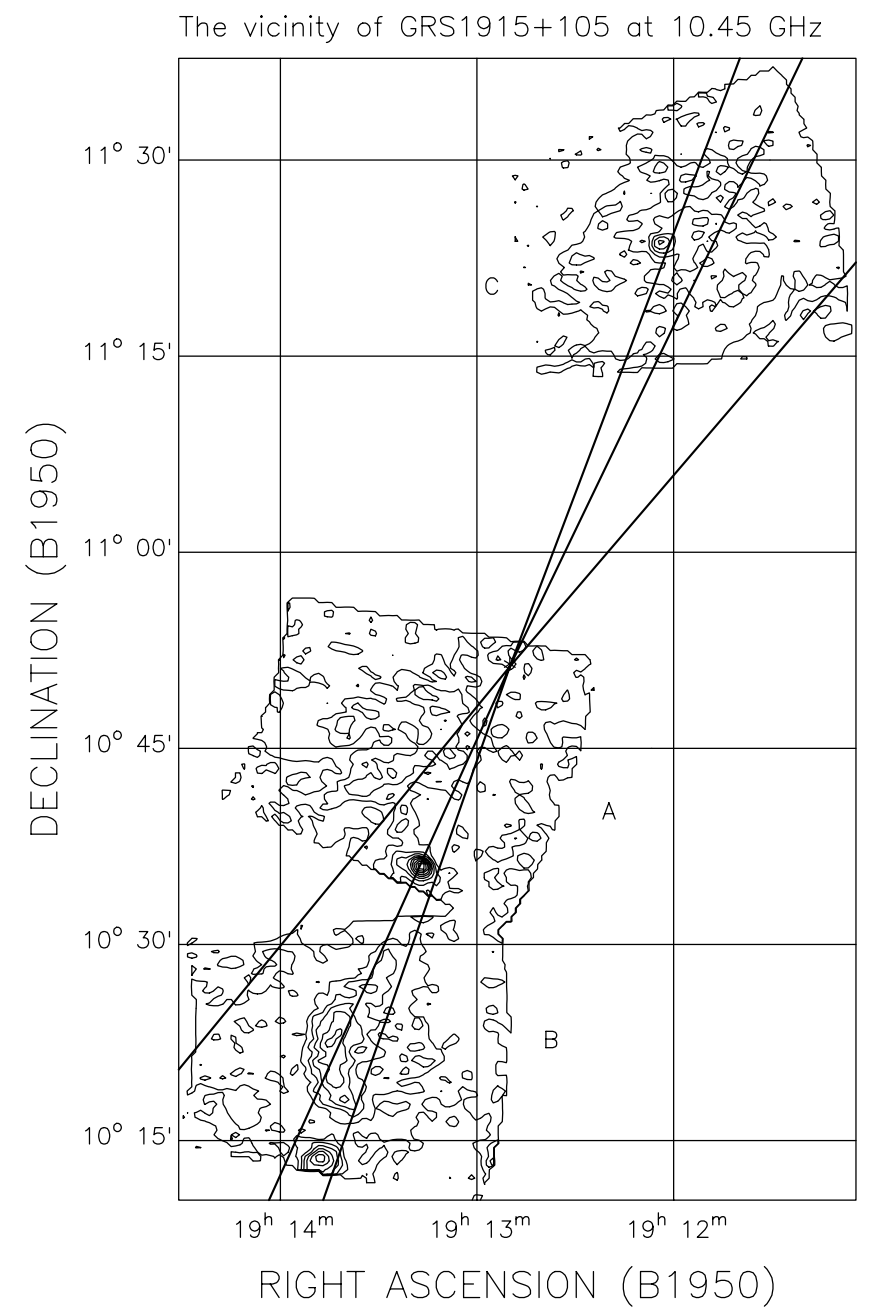

Fig. 2. The $10.45 \mathrm{GHz}$ Effelsberg maps of regions A, B and C from Fig. 1. The jet position lines given in the literature are plotted

\section{A review of the observed features}

All three objects found in the $11 \mathrm{~cm}$ data (Fig. 1) are also visible at $10.45 \mathrm{GHz}$. We used the method of differential spectral index plots (TT-plots, see Turtle et al. 1962) to obtain the temperature spectral index $\beta_{\mathrm{T}}\left(T_{\mathrm{B}} \propto \nu^{\beta_{\mathrm{T}}}\right)$ from the $11 \mathrm{~cm}$ survey data and the $10.45 \mathrm{GHz}$ observations. For the three objects, we obtained the following spectral indices:

Source A: $\quad \beta_{\mathrm{T}}=-2.41 \pm 0.2$

Source B: $\quad \beta_{\mathrm{T}}=-2.09 \pm 0.1$

Source C: $\quad \beta_{\mathrm{T}}=-2.03 \pm 0.1$.

Source A is a faint extended-emission plateau which could be of nonthermal nature. The integrated flux density is about $1.1 \mathrm{Jy}$. At the bottom of this area, a compact source of $99 \mathrm{mJy}$ is visible. It coincides with the infrared source IRAS $19132+1035$ discussed by RM98 and Chaty et al. (2000).

The sources in regions $\mathrm{B}$ and $\mathrm{C}$, outside the range observed by RM98, are very likely thermal emission features, probably faint HII emission. As seen in Fig. 1, the region B contains an extended "jet-like" feature, partly with a substantial thermal component. The present $10.45 \mathrm{GHz}$ observations reveal a shell-like morphology of this feature, characterized by a thermal-like spectrum. If this feature is produced by a jet-cloud interaction, the spectrum could be more complicated. In order to check this, additional observations at intermediate frequencies are required. The "hot-spot" at the bottom of Fig. 2 is characterized by a steeper non-thermal spectrum.

A map of region $\mathrm{C}$ at the top of Fig. 2 shows an extended source with a thermal-like spectrum. It is characterized by a complicated morphology with a number of brighter spots over the structure. We note that the brightest of these knots (visible also at the edge of the RM98 VLA map) is situated close to the axis formed by GRS 1915+105, the hot spot of region A (coincident with the IRAS 19132+1035, cf. RM98) and the previously discussed hot-spot in region B. The second IRAS 19124+1106 source studied by RM98 is situated outside fields observed by us, but also lies close to this axis.

\section{Conclusions}

Observations of a synchrotron nebula associated with a galactic source of relativistic jets would provide an opportunity to investigate processes of jet kinetic energy dissipation with much larger accuracy than in extragalactic sources. In particular, we are interested in observing the expected relativistic shock waves in jets' terminal points and/or interaction of such jets with molecular medium. Unfortunately, the position of GRS 1915+105 far away in the galactic plane, makes such observations difficult, due to effects of radiation attenuation, substantial depolarization effects at radio frequencies and superposition of many background/foreground sources occurring in the investigated region of the sky.

Our Effelsberg $10.45 \mathrm{GHz}$ observations performed within the $2^{\circ} \times 2^{\circ}$ region surrounding GRS $1915+105$ reveal a number of structures situated along the jets' direction, but - besides the positional coincidences - without any clear relation to GRS $1915+105$. We note three "hotspots" (plus the RM98 source IRAS 19124+1106) situated along the same axis of the position angle, approximately $160^{\circ}$. All three are the brightest points at the respective maps. The object coincident with the IRAS source discussed by RM98 shows a non-thermal extension along the jet direction. A possible identification of relativistic shocks at the mentioned hot spot positions could be done if substantial high energy photon fluxes are detected from these places. A preliminary review of existing X-ray observations did not reveal any such sources (M. BałucińskaChurch \& M. Church - private communication).

Acknowledgements. We are grateful to M. Urbanik and M. Soida for their help with preliminary reduction of the data and to M. Bałucińska-Church \& M. Church for review of the existing X-ray data and comments on the text. 
MO acknowledges support from the Komitet Badań Naukowych through the grants PB 179/P03/96/11 and PB 258/P03/99/17.

\section{References}

Beichmann, C. A. 1985, Infrared sources, catalogues, space observations, manuals, JPL, Pasadena

Chaty, S., Rodríguez, L. F., Mirabel, I. F., et al. 2000, A\&A, in press

Emerson, D. T., Klein, U., \& Haslam, C. G. T. 1979, A\&A, 76,120

Fender, R. P., Garrington, S. T., McKay, D. J., et al. 1999, MNRAS, 304, 865

Fender, R. P., \& Pooley, G. G. 1998, MNRAS, 300, 573

Fender, R. P., \& Pooley, G. G. 2000, MNRAS, 318, L1

Fürst, E., Reich, W., Reich, P., Honda, T., \& Sofue, Y. 1987, A\&AS, 69, 403

Ghisellini, G. 1999, in High Energy Processes in Accreting Black Holes, ed. J. Poutanen, \& R. Svensson, Graftavallen,
ASP Conf. Ser., 161, 249

Gliozzi, M., Bodo, G., \& Ghisellini, G. 1999, MNRAS, 303, L37

Levinson, A., \& Blandford, R. 1996, ApJL, 456, L29

Mirabel, I. F., \& Rodríguez, L. F. 1994, Nature, 371, 46

Mirabel, I. F., \& Rodríguez, L. F. 1999, ARA\&A, 37, 409

Morsi, H. M., \& Reich, W. 1988, A\&A, 163, 412

Hjellming, R. M. 1988, in Galactic and Extragalactic Radio Astronomy, ed. G. L. Verschuur \& K. I. Kellerman

Reich, W., Fürst, E., Haslam, C. G. T., Steffen, P., \& Reif, K. 1984, A\&AS, 58, 197

Rodríguez, L. F., \& Mirabel, I. F. 1998, A\&A, 340, 47

Rodríguez, L. F., \& Mirabel, I. F. 1999, ApJ, 511, 398

Sams, B. J., Eckart, A., \& Sunyaev, R. 1996, Nature, 382, 47

Schmidt, A., Wongsowjoto, S., Lochner, O., et al. 1993, Kleinheubacher Berichte, 36, 99

Turtle, A. J., Pugh, J. F., Kenderdine, S., \& Pauliny-Toth, I. I. K. 1962, MNRAS, 124, 459

Yadav, J. S., Rao, A. R., Agrawal, P. C., et al. 1999, ApJ, 517, 935 